\title{
Intrinsic Tumour Factors Affecting Recurrence in Non Muscle Invasive Bladder Cancer: a Hospital Based Study from India
}

\author{
Nisarg Mehta ${ }^{1}$, Ranjeet Singh Rathore ${ }^{1}$, Biju S Pillai ${ }^{1}$, Mohan P Sam ${ }^{1}$, Krishna \\ Moorthy $^{2 *}$
}

\begin{abstract}
Background: Depending on various pathological factors, non muscle invasive bladder cancer (NMIBC) shows varying degrees of recurrence. The aim of this study was to determine the incidence of recurrence of NMIBS in our centre, study the influence of intrinsic tumour characteristics like grade, stage, size and number, and compare our results with data in the published literature. Materials and Methods: A hospital based retrospective study was conducted on patients who underwent treatment for NMIBC from 2011 to 2014. The factors studied were number, size, grade, stage and site for correlation with recurrence. Statistical analysis was performed using Medcalc version 12, using Pearson's Chi square test to ascertain associations between variables. Results: A total of 73 patients with NMIBC were studied of which $48(65.8 \%)$ had low grade and $25(34.2 \%)$ had high grade tumours. Some 38 patients $(52.1 \%)$ had Ta tumours, 34 (46.6\%) had T1 and one had CIS. Mean follow up was 34.3 months. Recurrence rates were found to be $33.3 \%$ in low grade and $52.0 \%$ in high grade tumours. The overall recurrence rate in our centre was $39.7 \%$. Significant correlations were seen between stage and recurrence, with a rate of $15 \%$ for Ta and $63.3 \%$ for $\mathrm{T} 1$ tumours. Fourteen out of 21 bladder cancers $(66.6 \%)$ with multiple tumours demonstrated recurrence $(p=0.006)$. Grade, size and site had no influence. Conclusions: In our study, recurrence of NMIBC was found to be directly proportional to stage and number of primary tumours, but not grade, size and site. The incidence of recurrence of NMIBC both stage wise and grade wise in our centre was also low compared to the data in the published literature.
\end{abstract}

Keywords: Transitional cell carcinoma - NMIBC - recurrence - tumour factors

Asian Pac J Cancer Prev, 16 (7), 2675-2677

\section{Introduction}

The spectrum of primary malignancies of the urinary bladder can vary from superficial, well differentiated disease, which does not significantly impact patient survival, to highly malignant tumours for which long-term survival may be dismal. The vast majority of primary bladder tumours are of transitional cell variety (90-95\%). Squamous cell carcinoma (3-5\%) and adenocarcinoma $(1 \%)$ are found in a smaller subset of patients. Overall, bladder cancer is the seventh most common malignancy in men and the $17^{\text {th }}$ in women with the highest incidence seen at 70 years of age (Wu et al., 2008). Eighty percent of patients with bladder cancer are men (Jemal A et al., 2011).Recent trends indicate a small increase in the incidence of bladder cancer with an overall decline in mortality. Important risk factors reported for bladder cancer are chemical and environmental exposures, such as smoking and aromatic amines, and chronic irritation (Zeegers et al., 2000; Pelucchi et al., 2006).The primary classification of transitional cell carcinoma of bladder would be into superficial (non muscle invasive bladder cancer, NMIBC) and deep muscle infiltrating varieties. The disease characteristics and the management of these varieties are totally different. NMIBC however is notorious for its recurrence.

Though various studies have been done to identify the prognostic factors in the recurrence of superficial bladder cancer, studies on intrinsic tumour factors affecting the prognosis and recurrence of NMIBC are limited.

The aim of this study was to determine the incidence of recurrence of NMIBC in our hospital and to study the role of intrinsic pathological factors like number, grade, size and stage of tumour influencing recurrence and to compare the results with the data in published literature.

\section{Materials and Methods}

73patients with NMIBC were included in this hospital based retrospective study during the period $2011-2014$. TURBT was performed on these patients by qualified Urologists with more than 5 years of clinical experience. 
Patients with muscle invasive tumours on initial TUR biopsy, those with extensive growths, patients with upper tract tumours on initial presentation and those with metastases on initial presentation were excluded from the study.

TURBT was performed with a 26 Fr continuous flow resectoscope (monopolar). Sterile water was used as the standard irrigation fluid in all cases. The superficial and deep resection specimens were sent separately for histopathological studies.

The pathological factors studied in the tumours were number, size, grade, stage and site of tumour. Multiplicity was defined as tumours $\geq 3$ in number. Tumours more than $3 \mathrm{~cm}$ were considered as large tumours. Standard recommended dose of Intravesical BCG was given postoperatively in patients with high grade disease, T1 lesions and carcinoma in situ (CIS). Follow-up after TUR in these cases was done initially every 3 months for 2 years with cystoscopy and cytology, and every 6 months afterwards during the third to fifth years. Radiological investigations were done on annual basis. The patients were classified into low risk and high risk groups. Low grade, Ta tumours were considered low risk whereas tumours with high grade or T1 were classified under high risk group.

Statistical analysis was performed using Medcalc version 12 and p value less than 0.05 was considered statistically significant. Pearson's Chi-square test was done to ascertain the association between variables.

\section{Results}

Out of the 73 patients with NMIBC, 64 were males $(87.7 \%)$ and 9 were females $(12.3 \%)$. Incidence of tumours was maximum $(31.5 \%)$ in the age group of 61 to 70 years. History of smoking was found in 34 patients (46.6\%). Multiple tumours $(\geq 3)$ were seen in $21(28.8 \%)$ patients. Out of all the tumours, $48(65.8 \%)$ were of low grade and $25(34.2 \%)$ were of high grade. There were 38 patients $(52.1 \%)$ with Ta tumours, $34(46.6 \%)$ with T1 and one with CIS.

The location wise distribution of NMIBC in our centre is given in Table 1. Maximum numbers of tumours seen in our study were on the right lateral wall (28.7\%). Postoperative intravesical BCG in standard recommended dose was given in $43.8 \%$ of the patients. Mean follow up period was 34.3 months ( 3 months to 60 months). Overall recurrence of tumour was seen in $29(39.7 \%)$ patients out of which in $33.33 \%$ recurrence was seen in low grade and $52 \%$ in high grade tumours.

In the univariate analysis, significant association was seen between stage and recurrence $(\mathrm{p}=0.0007)$. Recurrence in Ta tumours was $18 \%$ and $61 \%$ in T1 tumours. 14 out of 21 bladder cancers with multiple tumours had recurrence $(\mathrm{p}=0.006)$. No significant association was seen between the site of tumour and the recurrence rate. Grade and size also had no influence on recurrence in our study. Out of the 23 cases of T1tumours, $13(56.5 \%)$ had early recurrence (<12 Months) whereas 9 out of $13(69.2 \%)$ high grade tumours showed recurrence within 1 year of follow up. These findings are depicted in Table 2.
Table 1. Location-wise Distribution of Tumours

\begin{tabular}{lr}
\hline Anterior Wall & 2 \\
Bladder Neck & 2 \\
Dome & 5 \\
Left Lateral Wall & 20 \\
Posterior Wall & 9 \\
Right Lateral Wall & 21 \\
Right Posterolateral Wall & 1 \\
Rt Lateral Wall,Bladder Neck, Posterior Wall & 1 \\
Trigone & 2 \\
Multiple Sites & 8 \\
\hline Total & 73 \\
\hline
\end{tabular}

Table 2. Recurrence of Various Tumours Based on Intrinsic Characters

\begin{tabular}{llccc}
\hline Intrinsic & Factors & Recurrence & Total number & $\%$ \\
\hline Grade & Low & 16 & 48 & 33.3 \\
& High & 13 & 25 & 52 \\
\multirow{2}{*}{ Stage } & Ta & 6 & 34 & 18 \\
& T1 & 23 & 38 & 61 \\
& TCIS & 0 & 1 & 0 \\
\multirow{4}{*}{ Size } & $<3 \mathrm{~cm}$ & 13 & 41 & 32 \\
\multirow{4}{*}{ Number } & $>3$ & 16 & 32 & 50 \\
& $>3$ & 15 & 52 & 29 \\
\hline
\end{tabular}

\section{Discussion}

The age and pattern of distribution of NMIBC in our centre was comparable to those reported in other centres worldwide with a marginal increase in the incidence in higher age group (Badar F et al., 2009; Yavari P et al., 2009).As in our study, smoking was found to be important associated factor in bladder cancer in other studies also(Badar F et al., 2009; Yaris F et al., 2006;Salehi A et al., 2011).Though Shakhssalim N et al. have reported the influence of various dietary factors on the incidence and recurrence of bladder cancer (Shakhssalim N et al., 2010), similar association could not be established in our series of patients, probably due to significant variation in the dietary menu in our population.

Though bladder cancer has a multi-factorial origin, various intrinsic factors have been found to determine the recurrence and progression of this disease. In the 2004 review of the European Association of Urology (EAU) guidelines, Oosterlinck had noted that multifocality was the most important tumour factor influencing recurrence and patients with $>6$ tumours at initial presentation had recurrence rate of virtually $100 \%$ (Oosterlinck W 2004). The second important factor that the author had predicted was the prior recurrence rate, calculated based on recurrence 3 months after TUR.

Millan-Rodriguez et al. analyzed the variables involved in recurrence in 1,529 patients with superficial carcinomas and concluded that the most important factors were multiplicity, size (greater than $3 \mathrm{~cm}$ ), presence of Tis and use of intravesical washes after TUR (that decreased the recurrence rate). Grade was not included among the factors studied (Millan-Rodriguez et al., 2000).

Regarding the time of recurrence, $51.7 \%$ of recurrent tumors in our series showed early recurrence. In the multivariate study, recurrence in the first 9 months was 
found to be an independent factor influencing recurrence (Millan-Rodriguez et al., 2000). Ali-El-Dein et al. (2003) also noted similar findings with recurrence in the first cystoscopy at 3 months being the only predictor for tumor progression, together with histological grade and DNA ploidy (Ali-El Dein et al., 2003).

With regard to site of tumour, there was no significantly higher recurrence rate for any particular site in our study. However, it is worth noting that tumours at the dome were associated with higher recurrence rates in some studies, which could be related to the technical difficulties involved in complete resection of tumours at this site during TURBT performed in these patients (Heney et al., 1982).

As regards histological grade of tumours, high grade tumors (G3) were reported to have recurrence of up to $80 \%$; however in our series high grade tumours recurred in only $52 \%$ cases (Herr, 2000).

Tumour stage represented another significant prognostic variable for NMIBC in various studies. Primary T1 tumours were found more frequently than Ta tumors and also showed higher rate of recurrence. A review of 2,410 patients with Ta and Tis from 7 EORTC phase III studies showed a trend to relapse ranging from $3.4 \%$ and $45.8 \%$ in the first cystoscopy performed after TUR of the bladder (Brausi et al., 2002). The stage or depth of tumour invasion in bladder wall appeared to make a difference in terms of prognosis in various series. Similar findings were observed in our study also.

Our study was conducted retrospectively with limited number of patients. Intravesical BCG could be a confounding factor while determining the recurrence rates; the rates may not be the actual recurrence for NMIBC when left untreated after TURBT. Recent developments, such as the use of various bio markers (Ghafouri-Fard $\mathrm{S}$ et al., 2014) and fluorescence cystoscopy, which are likely to identify early recurrence, were not undertaken in our study.

Though no mortality was seen in our series, Supit N et al interestingly reported that the overall survival of patients with NMIBC and MIBC was low in Indonesian population (Supit et al., 2011).

In conclusion, the recurrence of NMIBC was found to be proportional to stage and number of primary tumour in our centre. Grade, size and site of tumour did not have any influence on recurrence of tumour. The recurrence of NMIBC both stage-wise and grade-wise in our series of patients was less compared to the results given in published literature.

\section{References}

Ali-El-Dein B, Sarhan O, Hinev A, et al (2003). Superficial bladder tumours: Analysis of prognostic factors and construction of a predictive index. BJU Int, 92, 393-9.

Badar F, Sattar A, Meerza F, Irfan N, Siddiqui N (2009). Carcinoma of the urinary bladder in a tertiary care setting in a developing country. Asian Pac J Cancer Prev, 10, 449-2

Brausi M, Collette L, Kurth K, et al (2002). EORTC GenitoUrinary Tract Cancer Collaborative Group: Variability in the recurrence rate at first follow-up cystoscopy after TUR in stage TalT1 transitional Cell carcinoma of the bladder: a combined analysis of seven EORTC studies. Eur Urol, 41, 523-1.
Ghafouri-Fard S, Nekoohesh L, Motevaseli E (2014). Bladder cancer biomarkers: review and update. Asian Pac J Cancer Prev, 15, 2395-3.

Hall CM, Chang SS, Dalbagni G, et al (2007). Guideline for the management of nonmuscle invasive bladder cancer (Stages Ta, T1, and Tis): 2007 Update from the American Urological Association Education and Research, Inc J Urol, 178, 2314-0

Heney NM, Nocks BN, Daly JJ, et al (1982). Ta and T1 bladder cancer: location, recurrence and progression. $\mathrm{Br} \mathrm{J} \mathrm{Urol,}$ 52, $152-7$

Herr HW (2000). Tumor progression and survival of patients with high grade, noninvasive papillary (TaG3) bladder tumors: 15-year outcome. J Urol, 163, 60-1

Jemal A, Bray F, Center MM, et al. Global cancer statistics (2011). CA Cancer J Clin, 61, 69-0.

Millan-Rodriguez F, Chechile-Toniolo G, Salvador-Bayarri J, Palou J, Vicente-Rodriguez J. Multivariate analysis of the prognostic factors of primary superficial bladder cancer (2000). J Urol, 163, 73-8.

Oosterlinck W. Guidelines on diagnosis and treatment of superficial bladder cancer (2004). Minerva Urol Nephrol, 56, 65-2.

Pelucchi C, Bosetti C, Negri E, Malvezzi M, La Vecchia C (2006). Mechanisms of disease: the epidemiology of bladder cancer. Nat Clin Pract Urol, 3, 327-0.

Salehi A, Khezri A, Malekmakan L, Aminsharifi A (2011). Epidemiologic status of bladder cancer in Shiraz, Southern Iran. Asian Pac J Cancer Prev, 12, 1323-7.

Shakhssalim N, Hosseini SY, Basiri A, et al (2010). Prominent bladder cancer risk factors in Iran. Asian Pacific J Cancer Prev, 11, 601-6.

Supit W, Mochtar CA, Sugiono M, Umbas R (2011). Survival of patients with transitional cell carcinoma of the urinary bladder in Indonesia: A single institution review. Asian Pac J Cancer Prev, 12, 549-3

Sylvester RJ, Van der Meijden AP, Oosterlinck W, et al (2006). Predicting recurrence and progression in individual patients with stage Ta, T1 bladder cancer using EORTC risk tables: a combined analysis of 2596 patients from seven EORTC trials. Eur Urol, 3, 466-5.

Wu X, Ros MM, Gu J, Kiemeney L (2008). Epidemiology and genetic susceptibility to bladder cancer. BJU Int, 102, 1207-5.

Yaris F, Dikici MF, Sabuncu HH, Yaris E (2006). A case-control study on the etiology of urinary bladder cancer in Istanbul, Turkey. Asian Pac J Cancer Prev, 7, 591-4.

Yavari P, Sadrolhefazi B, Mohagheghi MA, Mehrazin R (2009). A descriptive retrospective study of bladder cancer at a hospital in Iran (1973-2003). Asian Pac J Cancer Prev, 10, 681-4.

Zeegers MP, Tan FE, Dorant E, van der Brandt PA (2000). The impact of characteristics of cigarette smoking on urinary tract cancer risk: A meta-analysis of epidemiologic studies. Cancer, 89, 630-9. 